\title{
Acquired Resistance to Isoniazid During Isoniazid Monotherapy in a Subject with Latent Infection Following Household Rifampicin-Resistant Tuberculosis Contact: A Case Report
}

\author{
Tsung-Lun $\mathrm{Li}^{1}$ \\ Tai-Hua Chan ${ }^{2}$ \\ Cheng-Hui Wang ${ }^{3-5}$ \\ Ruwen Jou ${ }^{2,6}$ \\ Ming-Chih $\mathrm{Yu}^{1,3,7}$ \\ Denise Utami Putri ${ }^{3}$ ** \\ Chih-Hsin Lee ${ }^{1,3,8, *}$ \\ Yi-Hsien Lin $^{3}$ \\ 'Department of Internal Medicine, \\ Wanfang Hospital, Taipei Medical \\ University, Taipei, Taiwan; ${ }^{2}$ Taiwan Centers \\ for Disease Control, Taipei, Taiwan; \\ ${ }^{3}$ Pulmonary Research Center, Wanfang \\ Hospital, Taipei Medical University, Taipei, \\ Taiwan; ${ }^{4}$ Department of Laboratory \\ Medicine, Wanfang Hospital, Taipei Medical \\ University, Taipei, Taiwan; ${ }^{5}$ School of \\ Medical Laboratory Science and \\ Biotechnology, College of Medical Science \\ and Technology, Taipei Medical University, \\ Taipei, Taiwan; 'Institute of Microbiology \\ and Immunology, National Yang-Ming Chiao \\ Tung University, Taipei, Taiwan; ${ }^{7}$ Schools of \\ Respiratory Therapy, College of Medicine, \\ Taipei Medical University, Taipei, Taiwan; \\ ${ }^{8}$ Division of Pulmonary Medicine, \\ Department of Internal Medicine, School of \\ Medicine, College of Medicine, Taipei \\ Medical University, Taipei, Taiwan \\ *These authors contributed equally to this \\ work
}

Correspondence: Chih-Hsin Lee; Denise Utami Putri

Pulmonary Research Center, Wanfang Hospital, Taipei Medical University, I I I Sec. 3, Xinglong Road Wenshan District, Taipei, I 16, Taiwan

Tel +886-2930-7930

Email chleetw@tmu.edu.tw; 108336@w. tmu.edu.tw

\begin{abstract}
Appropriate treatment is the key element in eliminating tuberculosis (TB), and requires prompt diagnosis. We presented a case of a household contact of rifampicin-resistant TB revealing reactive IFN-gamma release assay with unsuspicious clinical and radiologic examinations. She was diagnosed with latent tuberculosis infection (LTBI) and treated with isoniazid monotherapy. On the ninth month, she developed a progressive cough and was found to harbor active TB disease with added resistance to isoniazid. An individualized antiTB regimen consisting of moxifloxacin, kanamycin, prothionamide, ethambutol, and pyrazinamide was prescribed for 20 months, leading to sputum culture conversion and improvement of the reported symptom. No recurrence was observed on one-year follow-up. Assuming high compliance to therapy, we propose that the patient may have been underdiagnosed and received sub-optimal treatment leading to acquired-drug resistance. Conventional diagnosis methods based on immunological assay and radiographical findings may be insufficient to distinguish the incipient and subclinical states of TB from LTBI.
\end{abstract}

Keywords: latent tuberculosis infection, LTBI, treatment, acquired drug resistance, drugresistant $\mathrm{TB}$, subclinical $\mathrm{TB}$, case report

\section{Introduction}

Latent tuberculosis (TB) infection (LTBI) is "a state of persistent immune response to stimulation by Mycobacterium tuberculosis $(M t b)$ antigens with no evidence of clinically manifesting active TB", ${ }^{1}$ affecting at least $23 \%$ of the global population. ${ }^{2}$ With over 1.2 million deaths in $2018,{ }^{3}$ ultimate disease control necessitates successful treatment of both active and latent infection. ${ }^{1}$ Of those screened positive for LTBI, a lifetime risk of developing active TB is $5-10 \%$, especially during the first two years of TB exposure. ${ }^{1}$ Preventing this progression remains an urgent public health goal to reduce TB transmission. A large cohort study recently reported a rate of $0.28 \%$ active TB progression during or soon after LTBI treatment, while acquired drug resistance was not observed. ${ }^{4}$ Accurate diagnosis of the TB state is pivotal in treatment selection, yet, as TB disease lies within a wide clinical spectrum, ${ }^{5}$ apathetic use of prophylaxis in improperly targeted populations may arouse concern regarding potential side effects and acquired drug resistance.

The notification rate of TB in Taiwan was 37 per 100,000 population in $2019 .{ }^{6}$ Subjects who have contact history with index cases of culture-positive pulmonary 
TB are screened with chest radiography and interferongamma releasing assay (IGRA), and prophylactic treatment is advised for those diagnosed with LTBI. $^{7}$ The Taiwan National TB Program mandates directly observed treatment throughout the course of all anti-TB treatment. Here, we present the unique case of a subject with a history of contact with an index patient with rifampicinresistant TB. She was treated as an LTBI patient with isoniazid prophylaxis, but later developed active disease with resistance to both rifampicin and isoniazid, suggesting that the isoniazid resistance was acquired during the LTBI treatment. The Taipei Medical University Joint Institutional Review Board approved the study (No. N20200631) with patients' consent obtained.

\section{Case Description and Diagnostic Assessment}

A 29-year-old Taiwanese man presented to the outpatient clinic at the Taipei Municipal Wanfang Hospital, Taipei City, Taiwan, having experienced a chronic productive cough and yellowish sputum for five months. Prior consultations with local medical doctors left the symptoms unresolved. He reported frequent travel to Southeast Asian countries. On visit, chest auscultation revealed scattered bilateral coarse crepitations, which were more prominent on the right side; his physical findings were otherwise unremarkable. Chest radiography revealed a right upper lung cavity. The computed tomography scan showed multiple enhancement of lymphadenopathies in the mediastinum. Three sputum smears were negative for acid-fast bacilli (AFB); however, sputum culture was positive for Mtb. The GenoType MTBDRplus assay (Hain LifeScience GmBH, Nehren, Germany) revealed rifampicin resistance ( $r p o B$ gene H526D mutation) and susceptibility to isoniazid.

The patient was treated with moxifloxacin, kanamycin, isoniazid, ethambutol, and pyrazinamide. The treatment course was uneventful and sputum culture was converted to negative on the second month of treatment. The patient was cured after 20 months of treatment, and serial followups for two years showed no recurrence of TB (Figure 1).

His wife, a 36-year-old Vietnamese woman without underlying co-morbidities, underwent TB contact investigation. She reported no respiratory or constitutional symptoms. Physical examination revealed no abnormality, and previous medical history was unremarkable. The IGRA test was positive, and chest radiography was normal (Figure 2A), thus she was diagnosed with LTBI. She was prescribed nine months' isoniazid monotherapy $300 \mathrm{mg}$ once daily, directly observed by trained public health personnel. On the fourth month of treatment, she reported numbness and pain in her fingertips, which improved with vitamin B6 supplementation.

A progressive cough developed in the ninth month of treatment. She reported no fever, chills, hemoptysis, or night sweating. Chest auscultation was unremarkable, with a smooth breathing pattern and no lymphadenopathy. Physical examination was otherwise normal. Laboratory data on haemoglobin, blood count, liver and kidney function and laboratory tests were within normal results. Chest radiography revealed an opacity in the left upper lung (Figure 2B). Three consecutive sputum smear examinations were negative for AFB. The sputum GeneXpert test (Cepheid, California, United States) was positive for rifampicin-resistant $M t b$. The phenotypic drug susceptibility testing and GenoType MTBDRplus assay later confirmed resistance to isoniazid ( $k a t G$ deletion) and rifampicin (rpoB gene H526D mutation). Based on these findings, the patient was diagnosed as having active pulmonary TB with resistance to isoniazid and rifampicin. Further sequencing revealed susceptibility to fluoroquinolones, aminoglycosides, and pyrazinamide.

The wife was treated with an individualized anti-TB regimen comprising of moxifloxacin, kanamycin, prothionamide, ethambutol, and pyrazinamide, based on the results of the drug-susceptibility test. Following treatment, the sputum culture converted to negative after two weeks. Early on in the treatment course, she reported vertigo, blurred vision, pain on injection site, and gastrointestinal discomfort, thus ethambutol was later replaced with cycloserine. Following 20 months of anti-TB treatment, follow-up revealed no radiological or clinical evidence of recurrence up to a year later. Genotyping of the causative Mtb by the spacer oligonucleotide typing (spoligotyping) and Mycobacterial Interspersed Repetitive Units-Variable Number Tandem Repeats revealed that the $M t b$ strains from the two cases had identical genotypes.

\section{Discussion}

Growing cases of drug-resistant (DR)-TB raises concerns regarding the existence of inadequate or interrupted therapy in current clinical TB control practice. ${ }^{3}$ The gap between the discovery of new drugs ${ }^{8}$ and the emergence of resistance poses a major threat to the campaign to eliminate TB. Globally, $3.4 \%$ of new TB cases and $18 \%$ of previously treated cases had rifampicin-resistant tuberculosis. A recent global cross-sectional survey showed 


\begin{tabular}{|c|c|c|c|c|c|c|c|c|c|c|}
\hline \multirow[b]{3}{*}{$\begin{array}{c}\text { Patient } 1 \\
\text { (Husband) }\end{array}$} & \multirow{2}{*}{$\begin{array}{r}\text { First year } \\
\text { January }\end{array}$} & \multirow[b]{2}{*}{ February } & \multicolumn{5}{|c|}{ Second year } & \multicolumn{3}{|c|}{ Third year } \\
\hline & & & March & .. May ... & January & February & March & ... November & ... Septemb & aber ... \\
\hline & $\begin{array}{l}\text { Suspicion of TB } \\
\text { AFB smear (-) } \\
\text { Culture ongoing }\end{array}$ & $\begin{array}{l}\text { Culture (+) } \\
\text { Notify } \\
\text { Taiwan CDC }\end{array}$ & $\begin{array}{c}\text { Start RR-TB } \\
\text { treatment }\end{array}$ & $\begin{array}{l}2^{\text {nd }} \text { month } \\
\text { Culture } \\
\text { conversion }\end{array}$ & & & & Cured & & $\begin{array}{l}\text { Two years } \\
\text { follow-up }\end{array}$ \\
\hline \multicolumn{11}{|c|}{$\begin{array}{c}\text { Contact } \\
\text { investigation }\end{array}$} \\
\hline $\begin{array}{c}\text { Patient } 2 \\
\text { (Wife) }\end{array}$ & & & & $\begin{array}{l}\text { IGRA (+) } \\
\text { Start INH } \\
\text { treatment }\end{array}$ & $\begin{array}{l}9^{\text {th }} \text { month } \\
\text { progressive } \\
\text { cough }\end{array}$ & $\begin{array}{l}\text { Diagnosis of } \\
\text { MDR-TB } \\
\text { Start treatment }\end{array}$ & $\begin{array}{c}2^{\text {nd }} \text { week } \\
\text { culture } \\
\text { conversion }\end{array}$ & & Cured & $\begin{array}{l}\text { One year } \\
\text { follow-up }\end{array}$ \\
\hline
\end{tabular}

Figure I The timeline from the episodes of care of both patients.

Abbreviations: TB, Tuberculosis; AFB, acid-fast bacillus; CDC, Centers for Disease Control; RR, rifampicin-resistant; INH, isoniazid; MDR, multi-drug resistant; AE, adverse events.

isoniazid resistance rates of $7.4 \%$ and $11.4 \%$ for new and retreated cases, respectively. ${ }^{9}$ To minimize the risk of acquired drug resistance, treatment for active TB typically involves four or more antibiotics, while treating LTBI involves one or two antibiotics. ${ }^{1}$ This assumes a low risk of acquired resistance based on the belief of limited replication among the small number of viable bacteria in LTBI. $^{10}$ In the PREVENT TB trial investigating over 7000 high-risk subjects who underwent LTBI treatment, $0.19 \%$ and $0.43 \%$ of participants in the combination rifapentine-isoniazid and isoniazid-only groups, respectively, developed active TB. Isoniazid-resistant TB was identified in two patients from the isoniazid-only arm, and none from the combination rifapentine-isoniazid arm. ${ }^{11}$ A systematic review reported no definite risk of developing isoniazid resistance following isoniazid preventive therapy (relative risk $1.45,95 \%$ confidence interval $0.85-2.47$ ). ${ }^{12}$ While superinfection from later contact with index patients with DR-TB cannot be excluded, the risk of LTBI treatmentassociated acquired resistance was generally considered negligible. ${ }^{4}$ Additionally, mixed-strain $M t b$ infection may contribute to the occurrence of DR-TB. ${ }^{13}$

Currently, the IGRA test is considered the state-of-theart method for diagnosis of LTBI. However, IGRA assesses the T-cell response to TB antigens and cannot distinguish the latent infection from active disease. Reliable estimation of microbiological load is not available. Moreover, optimal diagnosis of active TB is also challenging as normal chest films are frequently reported, ${ }^{14}$ and interpretation of chest radiography results is often disputed by different observers. ${ }^{15}$ Negative mycobacterial culture results do not essentially exclude the possibility of active TB. Studies emphasized that the spectrum of human TB infection lies within the

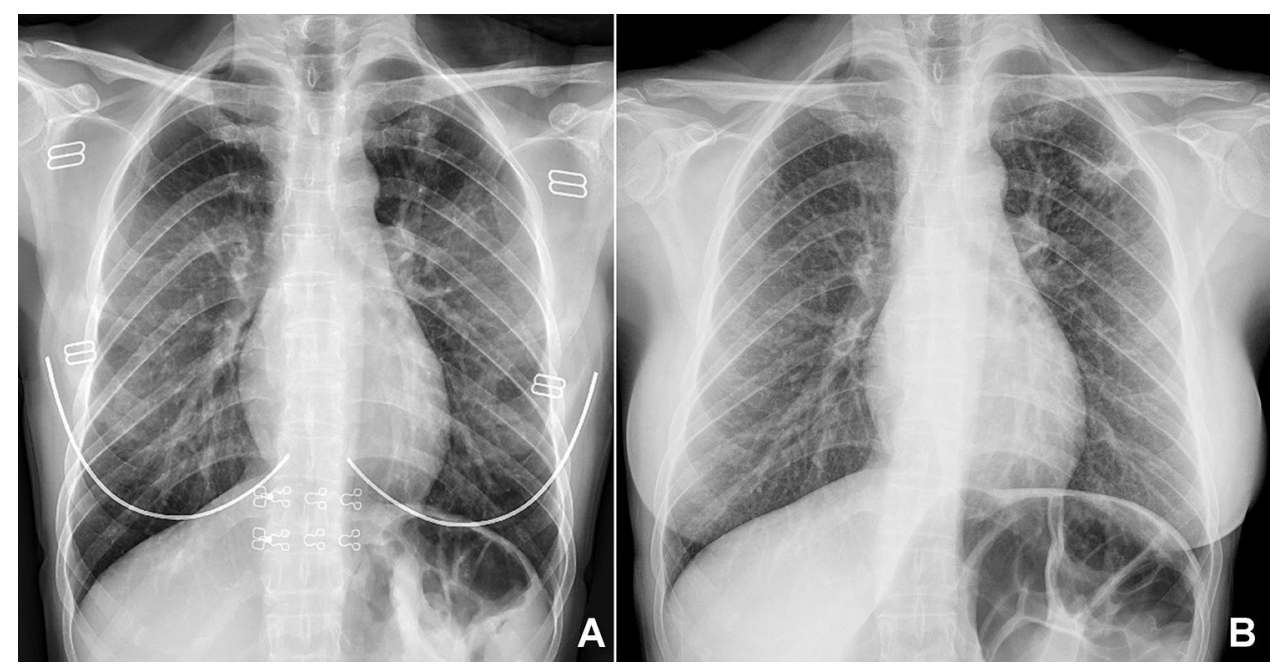

Figure 2 Chest radiography images $(\mathbf{A})$ before and $(\mathbf{B})$ after nine months of isoniazid treatment for the patient who progressed to active disease following the latent tuberculosis infection treatment. 
continuous dynamics of metabolic bacterial activities and hosts' immunological responses. ${ }^{16}$ Hence, in the early 20th century, further clinical staging of TB was introducedie, incipient and subclinical TB infection, which divided latent and active TB along the clinical disease spectrum. Briefly, both incipient and subclinical TB show a state of ongoing or impending progression of infection, but differ in the presence of radiographic abnormality or microbiological evidence of active replicating, viable $M t b$. Monotherapy for incipient TB has been proposed as an attractive alternative. However, the practical diagnostic criteria for incipient TB infection is not clearly defined, thus efficacy and risk of emergence of drug resistance for a specific regimen cannot be evaluated. ${ }^{5}$ Treating subclinical TB, which harbors active microbial replication and thus has a higher probability of resistance-associated mutations, with LTBI regimens theoretically bears a risk of emergence of resistant strains.

Given that incipient TB is not detectable on the basis of symptoms, radiography, or microbiological testing, our understanding of the epidemiology of incipient and subclinical TB is very limited at present. Definite evidence of the diagnosis of the incipient or subclinical TB state is unavailable in our case. Despite this, we pointed out an important occurrence of drug resistance following possible undiagnosed incipient/subclinical TB treated as LTBI. A detailed course of events and follow-up is presented, along with a comprehensive diagnostic approach to define the causative agent as well as the treatment regimen decision.

In summary, we presented a subject with contact history to an index patient of rifampicin-resistant pulmonary TB, who was diagnosed and treated as LTBI with isoniazid monotherapy on the basis of a positive IGRA test and no evidence of clinical nor radiological presentations. However, with a presumptive effective regimen and high compliance under a directly observed treatment program, her progression to active TB with acquired resistance to isoniazid was unexpected. In clinical practice, distinguishing incipient or subclinical TB infection from LTBI is unrealistic. In this case, we assume that the initial isoniazid treatment was "sub-optimal" and thus selectively intensified the acquired resistance to this drug when, at the time of contact screening, the wife would have been either at the incipient or subclinical TB states. While the possibility of mixed-strain infection cannot be excluded, the identical bacterial fingerprint observed from both patients renders this hypothesis less conclusive.

It is crucial to exclude subclinical or active TB during the management of LTBI to avoid acquired resistance. However, doing so is clinically challenging since reliable tools are still unavailable. The present report should raise concern regarding this issue and warrant future studies to develop more accurate testing methods, as well as diagnostic and therapeutic guidelines for further investigation of bacterial burden and clinical disease states.

\section{Patient Perspectives}

From the perspective of the husband, he was surprised and did not realize that there was still a risk of his wife developing TB even though his treatment was successful.

Now I know that the disease can spread before I was even diagnosed. I think the contact screening is important because I don't want any of my family to develop this disease again in the future.

From the perspective of the wife:

It is terrible to know that I got $\mathrm{TB}$ after the hard time completing nine months of medications. I did follow all the rules and have taken the drugs obediently! The doctors should have a better diagnostic tool to avoid what I experienced to happen to other people.

\section{Abbreviations}

AFB, acid-fast bacilli; IGRA, interferon-gamma releasing assay; LTBI, latent tuberculosis infection; $M t b$, Mycobacterium tuberculosis; TB, Tuberculosis.

\section{Data Sharing Statement}

The relevant data are included in the article. Further inquiries can be directed to the corresponding author.

\section{Ethics and Consent}

The studies involving human participants were reviewed and approved by Taipei Medical University Joint Institutional Review Board (No. N20200631). Patients provided written informed consent to have the case details and any accompanying images published.

\section{Author Contributions}

DUP and CHL made equal contributions and share correspondence. All authors contributed to data analysis, drafting or revising the article, have agreed on the journal to 
which the article will be submitted, gave final approval of the version to be published, and agree to be accountable for all aspects of the work.

\section{Funding}

No funding source was accepted for this study.

\section{Disclosure}

The authors declare that the research was conducted in the absence of any commercial or financial relationships that could be construed as a potential conflict of interest.

\section{References}

1. World Health Organization. Latent TB infection: updated and consolidated guidelines for programmatic management; 2018.

2. Houben RM, Dodd PJ. The global burden of latent tuberculosis infection: a re-estimation using mathematical modelling. PLoS Med 2016;13(10):e1002152. doi:10.1371/journal.pmed.1002152

3. World Health Organization. Global tuberculosis report 2019; 2019.

4. Flynn AG, Aiona K, Haas MK, Reves R, Belknap R. Clinical characteristics of active tuberculosis diagnosed after starting treatment for latent tuberculosis infection. Clin Infect Dis. 2019. doi:10.1093/cid/ ciz1157

5. Drain PK, Bajema KL, Dowdy D, et al. Incipient and subclinical tuberculosis: a clinical review of early stages and progression of infection. Clin Microbiol Rev. 2018;31(4):e00021-18. doi:10.1128/ CMR.00021-18

6. Taiwan Centers for Disease Control. Taiwan Tuberculosis Control Report 2019. 2020. Available from: https://www.cdc.gov.tw/File/Get/ eohpjs5F-9obJG4sMlmHBw. Accessed January 24, 2021.
7. Chiang C-Y. Taiwan Guidelines for TB Diagnosis \& Treatment. Vol. 6. Taiwan Centers for Disease Control; 2017.

8. Durairaj DR, Shanmughavel P. In silico drug design of thiolactomycin derivatives against Mtb-KasA enzyme to inhibit multidrug resistance of mycobacterium tuberculosis. Interdiscip Sci. 2019;11 (2):215-225. doi:10.1007/s12539-017-0257-0

9. Dean AS, Zignol M, Cabibbe AM, et al. Prevalence and genetic profiles of isoniazid resistance in tuberculosis patients: a multicountry analysis of cross-sectional data. PLoS Med. 2020;17 (1):e1003008. doi:10.1371/journal.pmed.1003008

10. Fox GJ, Dobler CC, Marais BJ, Denholm JT. Preventive therapy for latent tuberculosis infection-the promise and the challenges. Int J Infect Dis. 2017;56:68-76. doi:10.1016/j.ijid.2016.11.006

11. Sterling TR, Villarino ME, Borisov AS, et al. Three months of rifapentine and isoniazid for latent tuberculosis infection. $N$ Engl J Med. 2011;365(23):2155-2166. doi:10.1056/NEJMoa1104875

12. Balcells ME, Thomas SL, Godfrey-Faussett P, Grant AD. Isoniazid preventive therapy and risk for resistant tuberculosis. Emerg Infect Dis. 2006;12(5):744-751. doi:10.3201/eid1205.050681

13. Cohen T, van Helden PD, Wilson D, et al. Mixed-strain mycobacterium tuberculosis infections and the implications for tuberculosis treatment and control. Clin Microbiol Rev. 2012;25(4):708-719. doi:10.1128/CMR.00021-12

14. Mathur M, Badhan RK, Kumari S, Kaur N, Gupta S. Radiological manifestations of pulmonary tuberculosis - a Comparative Study between Immunocompromised and Immunocompetent Patients. J Clin Diagn Res. 2017;11(9):TC06-TC09. doi:10.7860/JCDR/ 2017/28183.10535

15. Eisenberg RL, Pollock NR. Low yield of chest radiography in a large tuberculosis screening program. Radiology. 2010;256(3):998-1004. doi:10.1148/radiol.10100485

16. Delogu G, Goletti D. The spectrum of tuberculosis infection: new perspectives in the era of biologics. $J$ Rheumatol Suppl. 2014;91:11-16. doi:10.3899/jrheum.140097
Infection and Drug Resistance

\section{Publish your work in this journal}

Infection and Drug Resistance is an international, peer-reviewed openaccess journal that focuses on the optimal treatment of infection (bacterial, fungal and viral) and the development and institution of preventive strategies to minimize the development and spread of resistance. The journal is specifically concerned with the epidemiology of antibiotic resistance and the mechanisms of resistance development and diffusion in both hospitals and the community. The manuscript management system is completely online and includes a very quick and fair peerreview system, which is all easy to use. Visit http://www.dovepress.com/ testimonials.php to read real quotes from published authors. 